\title{
ACCURATE OBJECT RECONSTRUCTION BY STATISTICAL MOMENTS
}

\author{
S P Prismall, M S Nixon and J N Carter \\ University of Southampton, United Kingdom
}

\begin{abstract}
Statistical moments can offer a powerful means for object description in object sequences. Moments used in this way provide a description of the changing shape of the object with time. Using these descriptions to predict temporal views of the object requires efficient and accurate reconstruction of the object from a limited set of moments, but accurate reconstruction from moments has as yet received only limited attention. We show how we can improve accuracy not only by consideration of formulation, but also by a new adaptive thresholding technique that removes one parameter needed in reconstruction. Both approaches are equally applicable for Legendre and other orthogonal moments to improve accuracy in reconstruction.
\end{abstract}

\section{INTRODUCTION}

The value of statistical moments in computer vision problems has been long established, the field having been pioneered by $\mathrm{Hu}$ [2] in the early 1960's. Almost all the literature on moments in computer vision/image analysis has been concerned with static images. However, in recent years there has been a growing interest in image sequences and this has led to the development of velocity moments [6] where moments are used in space and time simultaneously. Our motivation lies in using the change in moment values through an object sequence to construct novel temporal object views [5]. This has led to investigation into the reconstruction properties of various types of moments.

Statistical moments offer ability to select differing levels of detail. In addition, the completeness of their description results in one of their often cited attributes, the ability to reconstruct an object from its set of moments. However, most research with moments has concentrated on their descriptive properties, while the (few) studies that have considered reconstruction have tended to approach reconstruction to validate recognition, rather than explore the accuracy of the reconstruction. The emphasis on recognition has motivated a lack of consideration of the accuracy of reconstruction. Given that we seek to be able to interpolate or predict the appearance of an object from its feature description (as such, not to predict the complete image containing the object), we sought to investigate the basic processes of reconstruction and found new ways in which its accuracy can be improved. Of particular interest here, is a threshold parameter that can affect performance. By obviating this parameter, performance can be enhanced.

Section 2 shows how the orthonormality of Legendre moments can be properly achieved. This resolves earlier difficulty in moment reconstruction, allowing for extension to be made. We then describe a new approach to binary object reconstruction by moments which is achieved by adaptive means and then show in Section 3 how our new approaches can lead to accurate object reconstruction. Section 4 draws conclusions from this work and sets out areas requiring further investigation.

\section{OBJECT RECONSTRUCTION FROM ORTHOGONAL (ORTHONORMAL) MOMENTS}

The image moments can be calculated generically by:

$$
\phi_{p q}=\iint_{\zeta} \psi_{p q}(x, y) f(x, y) d x d y
$$

which defines a moment $\phi$ of order $(p+q)$ over an image in the region $\zeta$, with the function $\psi(x, y)$ known as the basis set. In its simplest form the basis set is the monomial $\left\{x^{p} y^{q}\right\}$ giving rise to geometric moments, $m_{p q}$. The uniqueness theorem states that the moment set $m_{p q}$ is unique for a given image function $f(x, y)$. In addition, the existence theorem states that the moments of all orders exist [4]. These two theorems give rise to the reconstruction property of moments.

\subsection{Moments and Reconstruction}

Reconstruction from geometric moments (and other non-orthogonal types) is not trivial and requires moment matching [7]. Reconstruction from orthogonal moments is much less demanding. The Legendre moments of an image can be defined as:

$$
\lambda_{m n}=k \int_{-1}^{1} \int_{-1}^{1} P_{m}(x) P_{n}(y) f(x, y) d x d y
$$

where $(m+n)$ is the order (as with geometric moments), $k$ is a normalisation constant and the Legendre polynomial of variable $x(-1 \leq x \leq 1)$ and order $n$ is: 


$$
P_{0}(x)=1, \quad P_{n}(x)=\frac{1}{2^{n} n !} \frac{d^{n}\left(x^{2}-1\right)^{n}}{d x^{n}} \quad n=1,2, \cdots
$$

Much of the literature suggests that the orthogonality of the basis functions allows for easy reconstruction of the original function. In reality this is only partially true since the basis functions should be orthonormal (i.e. orthogonal and normalised). However most derivations of orthogonal moments described in the literature (e.g. in [7]) are not based on orthonormal polynomials. However, the normalisation that is used in the calculation of the moments allows for correct reconstruction - it could be argued that correct normalisation is achieved over the two transforms. In this section the correct normalisation of orthogonal moments is discussed and consideration given to normalisation in discrete systems.

\subsection{Legendre moments}

The Legendre moments use Legendre polynomials as the basis functions. The usual definition given for the Legendre moment (as given by Teague [7]) is:

$$
\lambda_{m n}=\frac{(2 m+1)}{2} \frac{(2 n+1)}{2} \int_{-1}^{1} \int_{-1}^{1} P_{m}(x) P_{n}(y) f(x, y) d x d y
$$

Note that the Legendre polynomials are orthogonal over the range $-1 \leq x \leq 1$, and that the factors outside of the integral are for normalisation of the Legendre moments. However, it should be noted that the Legendre polynomials are not normalised (i.e. they are orthogonal, but not orthonormal). The orthogonality condition for the Legendre polynomial is [1]:

$$
\int_{-1}^{1} P_{m}(x) P_{n}(x) \quad d x=\frac{2}{2 n+1} \delta_{m n}
$$

where $\delta_{m n}$ is the dirac delta function. Thus,

$$
\int_{-1}^{1}\left(\sqrt{\frac{2 n+1}{2}} P_{n}(x)\right)\left(\sqrt{\frac{2 n+1}{2}} P_{n}(x)\right) d x=1
$$

giving the orthonormal Legendre polynomial, $\hat{P}_{n}(x)$ :

$$
\hat{P}_{n}(x)=\sqrt{\frac{2 n+1}{2}} P_{n}(x)
$$

From this, we can define the orthonormal Legendre moment, $\lambda_{m n}$, as:

$$
\lambda_{m n}=\int_{-1}^{1} \int_{-1}^{1} \hat{P}_{m}(x) \hat{P}_{n}(y) f(x, y) d x d y
$$

or, from unnormalised Legendre polynomials:

$$
\lambda_{m n}=\sqrt{\frac{2 m+1}{2}} \sqrt{\frac{2 n+1}{2}} \int_{-1}^{1} \int_{-1}^{1} P_{m}(x) P_{n}(y) f(x, y) d x d y
$$

A comparison of equation (9) with (4) shows that the previous definition of the Legendre moment is not orthonormal. At this point, it is interesting to consider the reconstruction of functions from its moments. The (re)construction of a function, $g(x, y)$, from a set of Legendre moments up to and including order $M$ of a function $f(x, y)$ is usually reported as (from [7]):

$$
g(x, y)=\sum_{m=0}^{M} \sum_{n=0}^{M-m} \lambda_{m n} P_{m}(x) P_{n}(y)
$$

However, our orthonormal Legendre moments, $\lambda_{m n}$, are effectively derived from the orthonormal Legendre polynomials, $\hat{P}_{n}(x)$ (see equations (7) and (8)). Hence, for orthonormal moments the reconstruction formula should be written as:

$$
g(x, y)=\sum_{m=0}^{M} \sum_{n=0}^{M-m} \lambda_{m n} \hat{P}_{m}(x) \hat{P}_{n}(y)
$$

or (using unnormalised Legendre polynomials):

$$
g(x, y)=\sum_{m=0}^{M} \sum_{n=0}^{M-m} \lambda_{m n} \sqrt{\frac{2 m+1}{2}} P_{m}(x) \sqrt{\frac{2 n+1}{2}} P_{n}(y)
$$

It is important to note here that calculating the Legendre moments by equation (4) (i.e. they are not actually orthonormal), then reconstructing with (10) will give the same function as that using equation (11) (or (12)) with the orthonormal Legendre moments from equation (8) (or (9)). Therefore, although the previously reported analyses are not orthonormal, the resulting reconstructions have actually been correct, i.e. the normality has been achieved across the two transforms. A similar situation is to be found with Zernike moments. Clearly, if the aim of reconstruction is to validate recognition capability, this appears to be of little consequence. However, if there is purpose to the reconstruction or if the moment values are used in conjunction with each other, then this mandates the use of this corrected analysis.

\subsection{Adaptive Reconstruction of Binary Images}

The reconstruction of a function from a limited set of orthogonal moments is easily effected by application of equation (11) (for Legendre moments), and in the limit with an infinite set of moments, the reconstructed function will match the original function. The reconstruction of a function can be seen as a summation of weighted (by the moment value) basis functions. Since the basis functions are continuous functions, it is clear that the reconstructed function will also be continuous. However, in the case of computer images, the intensity values are discrete. Thus any representation of the reconstructed function needs to be approximated to the discrete values.

In the case of a binary object, this approximation can be achieved by using an appropriate threshold on the reconstructed function. In reality, the selection of an appropriate threshold has received very little attention and is usually set at the mid-point between the minimum and maximum values of the reconstructed function, e.g. in [3]. This is perhaps intuitive, and indeed, recent work [5] has confirmed that this threshold performs well (in terms of error measured 
against the original binary image) as a generic value in the absence of other information. However, there is information that can be used to steer the threshold selection. The zero-order moment for any object gives the mass of the object, and in the case of a binary object, a count of the number of pixels in it. Therefore, as long as the zero-order moment is known, then its value can be used to set an appropriate threshold such that the mass of the reconstructed function is the same as the original function. This technique of threshold selection will be referred to as adaptive thresholding. It will be shown later that the use of adaptive thresholding leads to a more accurate object reconstruction especially at lower orders.

The question of accuracy of reconstruction leads to the question as to how this is measured. Previous work such as $[3,5]$ has adopted the approach of counting the errors between the two objects. While this obviously gives a measure of how many pixels are incorrect, it does not give a feel for how close two objects are to matching. This is illustrated in Figure 1. Figure 1a shows part of an original object, while Figure $1 \mathrm{~b}$ and $1 \mathrm{c}$ show similar objects. By merely counting different pixels, the two objects are each as accurate as a representation of the original, although intuitively Figure $1 \mathrm{~b}$ would appear to be more accurate.

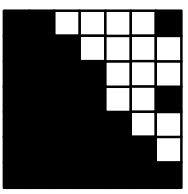

a)

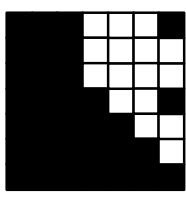

b)

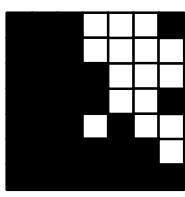

c)
Figure 1: Part of a binary object and similar objects.

In order to take account of the pixel distribution, we propose a simple error metric, whereby an incorrect pixel is weighted by the square of the distance to the nearest correct pixel. The weighted error, $W$, of the reconstruction, with $i$ error pixels at a distance $r$ from the nearest correct pixel is given by:

$$
W=\sum_{i} r_{i}^{2}
$$

The use of the square of the distance has obvious comparisons with the variance in statistics.

\section{RECONSTRUCTION ANALYSIS}

Results were developed for reconstructing $64 \times 64$ binary objects, of which examples are shown in Figure 2.

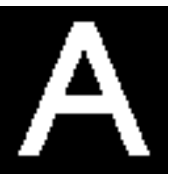

a)

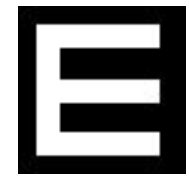

b)

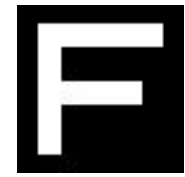

c)

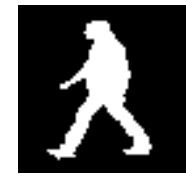

d)
Figure 2: Various test binary objects.

Table 1 shows some specific moment values for the binary ' $A$ ' shown in Figure $2 a$ with varying degrees of normalisation (the correct normalisation from equation 9, and normalisation used in [7]). In addition, Table 1 also shows the relative magnitudes of the moments. This demonstrates that if the moments are used in relation to each other, then the correct normalisation is vital since the relative magnitude for the corrected moments differs greatly from their previous uncorrected versions.

\begin{tabular}{|c|c|c|c|c|}
\hline \multicolumn{2}{|c|}{$\begin{array}{c}\text { TABLE 1: Some Legendre moment values for the letter A using } \\
\text { various degrees of normalisation. }\end{array}$} & $\begin{array}{c}\text { Relative } \\
\text { magnitude } \\
\text { normalised as } \\
\text { eqn. (9) }\end{array}$ & $\begin{array}{c}\text { Relative } \\
\text { magnitude } \\
\text { compared } \\
\text { with } \lambda_{00}\end{array}$ & $\begin{array}{c}\text { Normalised } \\
\text { as in [7] } \\
\text { compared with } \\
\lambda_{00}\end{array}$ \\
\hline$\lambda_{00}$ & 0.220 & 1.000 & 0.110 & 1.000 \\
\hline$\lambda_{21}$ & 0.047 & 0.215 & 0.058 & 0.527 \\
\hline$\lambda_{40}$ & 0.014 & 0.064 & 0.016 & 0.143 \\
\hline$\lambda_{65}$ & 0.013 & 0.060 & 0.043 & 0.390 \\
\hline$\lambda_{25}$ & -0.030 & -0.137 & -0.064 & -0.584 \\
\hline
\end{tabular}

It is well known that increasing moment orders capture increasing detail, so while the basic shape of an object can be reconstructed from relatively few low-order moments, the accuracy of the detail requires the inclusion of higher-order moments in the reconstruction set. Figure 3 shows the reconstruction of the letter A, using an increasingly large moment sets. The thresholded reconstructions are shown, using the conventional threshold of 0.5 and also the adaptive threshold described in Section 2.3.

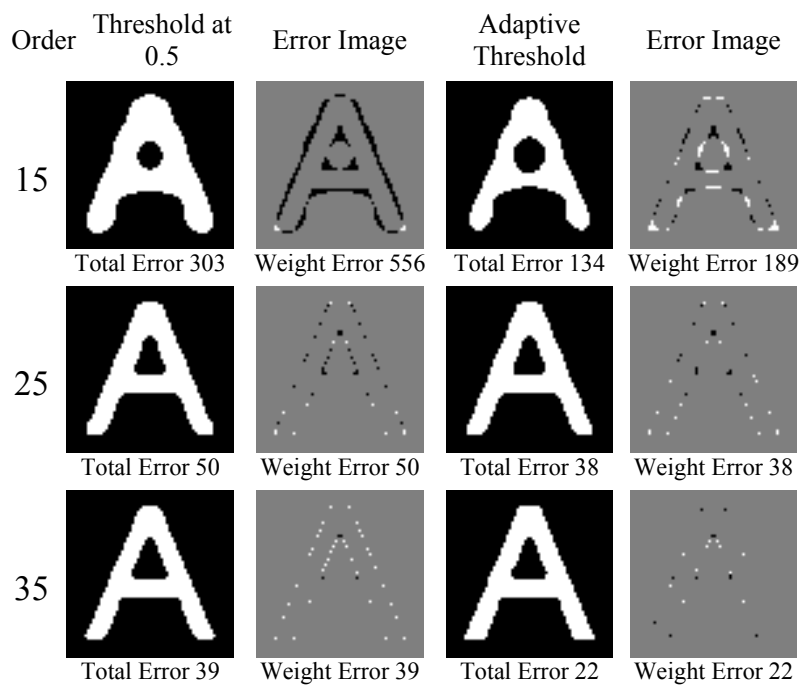

Figure 3: Example reconstructions at various orders.

It is clear in Figure 3 that the adaptive threshold performs better than a fixed threshold, especially at lower orders. Note that order 15 implies 136 moments, which is perhaps larger than the number of moments often used for recognition. In particular, there appears to be a continual improvement in the reconstruction quality with increasing order using the adaptive threshold, demonstrating the extra detail being added with each order, while with the fixed threshold, this overall improvement is more random. It is also worth noting that the error pixels have a structured appearance with the fixed threshold while the errors with the adaptive threshold have a more random (and less 


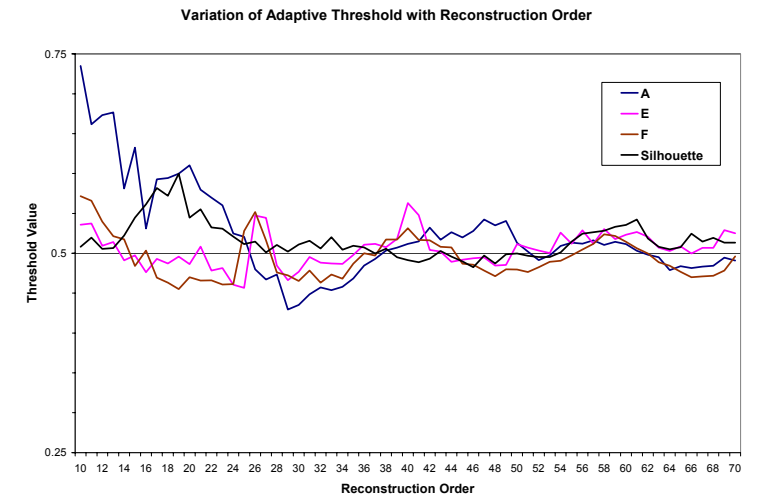

Figure 4: Variation in adaptive threshold with the reconstruction order for the various original objects.

grouped) appearance (for example, see order 35 in Figure 3).

It is obvious that as the reconstruction becomes better (i.e. more 'black' and 'white'), then the selection of the threshold becomes less critical, and indeed this has already been seen in [5], where different thresholds were seen to offer reasonable performance at higher orders. Figure 4 shows how the value of the adaptive threshold changes with reconstruction order. The adaptive thresholds quickly tend towards 0.5 as the order increases, but at lower orders there can be a substantial difference between the adaptive threshold and the fixed 'ideal' threshold of 0.5. This difference accounts for the improved reconstruction performance when using the adaptive threshold.

Figure 5 demonstrates how the weighting of error pixels that was discussed in Section 2.3 influences the degree of error found in the reconstruction. It is clear from this graph that as the reconstruction error increases so the degree of the error diminishes, and also as the error pixels become more diffuse the weighted error coalesces with the total error (in terms of the number of incorrect pixels). However, it is clearly shown that at lower orders where there is more grouping of the error pixels, the weighted error is significantly higher which reflects the intuitive situation of diffuse errors being less significant (or noticeable) than highly grouped errors.

Figure 5 also shows that in the case of the silhouette (Figure 2d) the reconstruction are significantly less

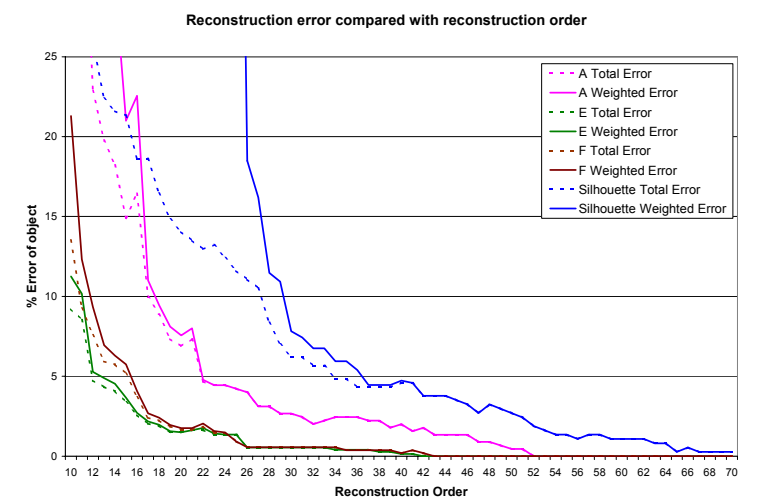

Figure 5: Graph of reconstruction error with order (weighted and total). accurate. This is in part due to the object area of the silhouette being significantly smaller than that of the other objects, but is really a reflection of the greater amount of detail in this 'real' object (i.e. derived from real data) over the synthetic ones. At order 35 (a total of 666 moments), the \% weighted error of the silhouette is $5.9 \%$ (or $1.1 \%$ of the whole image) a total of 36 pixels (44 when weighted). This shows that even with a complex real object, the reconstruction is very accurate. This is significant as we wish to extend this work to look at reconstruction of real objects within temporal sequences.

\section{CONCLUSIONS}

We have shown that accurate reconstruction of binary objects can be achieved using a limited set of Legendre moments, and in particular, how selecting a threshold guided by the zero-order moment of the object can improve the binary representation of the reconstructed function. This use of an adaptive threshold is equally valid when using Zernike moments. While the set of moments used (at order 35, there are 666 components) does not represent an especially compact description of the object, it is a description that can be used temporally and as such is of benefit for use in the description of moving (articulated) objects.

In future we will apply the reconstruction techniques to moment sets that have been predicted from a temporal object sequence, allowing temporal object construction at any point in the sequence.

Acknowledgements. We gratefully acknowledge partial support by the European Research Office of the US Army under Contract No. N68171-01-C-9002. We also grateful to Dr S Weiss for advice and suggestions.

\section{REFERENCES}

1. Courant R., and Hilbert D., 1953, "Methods of Mathematical Physics, Volume I", Interscience, New York.

2. $\mathrm{Hu}$ M.K., 1962, "Visual pattern recognition by moment invariants", IRE Trans. Info. Theory, IT-8, 179-187.

3. Khotanzad A., and. Hong Y.H., 1990, "Invariant image recognition by Zernike moments", IEEE Trans. PAMI, 12(5), 489497.

4. Mukundan R., and Ramakrishnan K.R., 1998, "Moment functions in image analysis: theory and applications", World Scientific, Singapore.

5. Prismall S.P., Nixon M.S., and Carter J.N., 2002, "On moving object reconstruction by moments", BMVC 2002, 73-82.

6. Shutler J.D., and Nixon M.S., 2001, “Zernike velocity moments for description and recognition of moving shapes", BMVC 2001, 705714.

7. Teague M.R., 1979, "Image analysis via the general theory of moments", Journal of the Optical Society of America, 70(8), 920-930.

8. Teh C.-H., and Chin R.T., 1988, "On image analysis by the method of moments",. IEEE Trans. PAMI, 10(4), 496-513. 


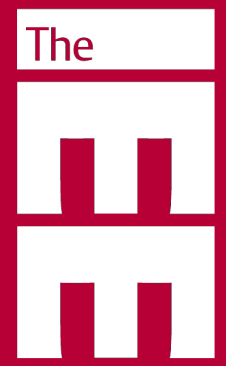

Click to return to session page

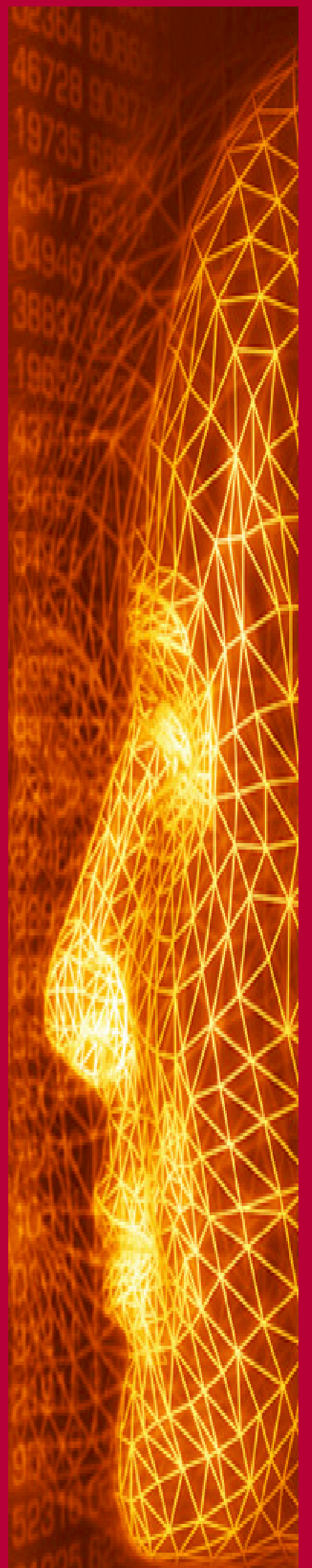

